\title{
Cooperative Spectrum Sensing by Algorithmic Techniques Using Spatial Information
}

\author{
Taha Abachi, Mohammad Afshin Hemmatyar, Mohammad Amin Fazli, Mohammad Izadi \\ CE Department, Sharif University of Technology, Tehran, Iran \\ E-mail: abachi,fazli@ce.sharif.edu, hemmatyar, izadi@sharif.edu
}

\begin{abstract}
Spectrum sensing is the basic step in cognitive radio networks. Its accuracy directly affects spectrum utilization. In this paper we propose a new method for blind cooperative spectrum sensing using spatial information. We model our sensing matrix as a graph, taking advantage of signal and spatial correlation of antennas. We prove that the general settings of the problem are hard to approximate. Taking spatial information into account, we propose a new scheme to find white space subregions. Cognitive radio users residing in the region may be able to transmit, but must keep their transmission power to the extent that no interference is caused to primary receivers out of the region.
\end{abstract}

Index Terms-Cognitive Radio, Cooperative Spectrum Sensing, Approximation Algorithm, Clique, Convex Hull

\section{INTRODUCTION}

The concept of the cognitive radio was conceived based on the FCC Task Force report [1] in 2004 on the underutilization of the wireless radio environment. According to the report primary users did not used their licensed spectrum from 15 to $85 \%$ time. Therefore these silence ranges in time and frequency can be exploited by unlicensed users. The only point these users should keep in mind is not to cause any kind of interference to primary users. The cognitive radio was first defined by Joseph Mitola [2] as follows: A cognitive radio is capable of reconfiguring its working parameters based on the surrounding environment conditions.

As the definition offers the CR should have these two capabilities [3]:

- Cognition: It is obvious that the CR must be able to sense and measure the spectrum in order to adapt the working parameters. Then the information gathered about the working environment is used to calculate new working parameters such as frequency, power, data rate and even Direction-Of-Arrival (DOA).

- Reconfiguration: The CR should be able to set the new parameters as working ones. So it should be able to reconfigure them either in hardware or software.

The cognition cycle includes four steps [4]: spectrum sensing, spectrum decision, spectrum sharing and mobility. During the sensing step all the available spectrum is searched for the presence of primary users and a spectrum map is drawn. The map is used to decide the best available spectrum for the secondary users based on the Quality-Of-Service requirements in the spectrum decision step. Once the best spectrum is selected, the secondary users can share the spectrum. The sharing scheme should be fair to avoid malicious (selfish) users. When a primary user shows up in the channel or the channel characteristics degrade to the level unacceptable for secondary users, a new channel should be selected. The spectrum mobility step deals with keeping a connection seamless.

The works by Cabric et al [6], Akyildiz et al [3]. and Haykin [5] provide a summary of these classical techniques from the cognitive network point of view. It is clear from these works, that none can fully cope with all the requirements of the cognitive radio networks.

In this work we propose a new method for blind (as no a priori knowledge is needed) cooperative spectrum sensing that is able to find subregions that do not receive primary signals and are suitable for cognitive radio transmission that resides inside the subregion. The method relies on the signal received by multiple randomly distributed antennas and their spatial correlation defined as their locality factor.

\section{Some Algorithmic Preliminaries}

This section includes some algorithmic concepts that are important for understanding the results in this paper. The first concept, we are supposed to introduce is the concept of approximation algorithms. Approximation algorithms are the most important approach for solving NP-Complete problems. As you know there is no polynomial time algorithm for NPComplete problems. The idea behind these kind of algorithms is to lessen the degree of problem tightness and solve the new problem with an algorithm in polynomial time which guarantees the answer to be in an efficient distance from the optimum answer. Our problem is a maximization problem. So for simplicity, we define the concepts of this part in the context of maximization problems.

DEFINITION 2.1: We call an algorithm $A$ for a maximization problem $P$ an Approximation Algorithm if:

- Its solution is a feasible solution for our problem.

- there is a real number $\epsilon$ so that for every instance $I$ of $P$ we have $A(I) \geq(1-\epsilon) . O P T(I) . A(I)$ is the output of the algorithm $A$ on input $I . O P T(I)$ is the optimal solution for this input. We call the parameter $1-\epsilon$, the factor of the algorithm.

Approximation algorithms are important because they can support an answer that is a good estimation of the optimal answer. They can also be used as a heuristic for the local search algorithms.

In this paper we need algorithmic results related to two important NP-Complete problems: 
DEFINITION 2.2: In problem MAX-CLIQUE, we are given a graph $G$ and we want to find the maximum complete subgraph of $G$ called clique, means that we want to find a subgraph of $G$ in which every two vertices are connected via an edge and also has the maximum number of vertices.

DEFINITION 2.3: In problem MIN-CLIQUEPARTITION we are given a graph $G$ and the problem is that of partitioning the vertex set of a given graph into a minimum number of cliques.

For general graphs, we know that MAX-CLIQUE is inapproximable within factor $n^{1-\epsilon}$, for any $\epsilon>0$, unless $P=N P$.

Theorem 2.4: [10] For any $\epsilon>0$ there is no approximation algorithm with factor better than $n^{1-\epsilon}$ for MAX-CLIQUE problem.

There is a similar inapproximability result for MINCLIQUE-PARTITION problem. For general graphs, MINCLIQUE-PARTITION is equivalent to minimum graph coloring of the complement graph, which is known to be inapproximable within $n^{1-\epsilon}$, for any $\epsilon>0$, unless P=NP.

Theorem 2.5: [16] For any $\epsilon>0$ there is no approximation algorithm with factor better than $n^{1-\epsilon}$ for MIN-CLIQUEPARTITION problem.

We will use these results to prove that the general setting of our problem is very hard to deal and not approximable. After considering our problem from the complexity view, we will take spatial information into account and create a new model in which some special kind of graph will be taken as the main object. The following definition describes them:

DEFINITION 2.6: Unit Disk Graphs(UDG) are graphs in which each vertex(a point in 2D space) is connected to all vertices that reside in a disk with radius $r$ around it. In this paper we call $r$, the UDG threshold.

MAX-CLIQUE problem can be solved in polynomial time in UDGs:

Theorem 2.7: [13] There is a polynomial time algorithm solving MAX-CLIQUE problem in UDGs.

But MIN-CLIQUE-PARTITION still remains NP-Complete in these kind of graphs [17]. Capolyleas et al [15] proved an important property about the optimal answers of this problem:

Theorem 2.8: [15] Given a finite point set $S$ in the plane, there exists an optimal clique partition where the convex hulls of the cliques are non-overlapping.

If you are not familiar with convex hull term you can refer to following definition:

Definition 2.9: The Convex Hull of a set $S$ of points is a convex polygon that every point of $S$ resides in it or on the perimeter of it.

There are many algorithms proposed for finding the convex hull of a given set of points that operates in $O(n \cdot \log (n))$. You can find some of them at [18]. The most famous one is Graham Scan Algorithm.

The theorem 2.8 is important because it simplified developing algorithms for the problem by bounding the answer domain. Pirwani et al in [11] proposed a PTAS for this problem. PTAS is a kind of approximation algorithms with dynamic factor and dynamic running time.

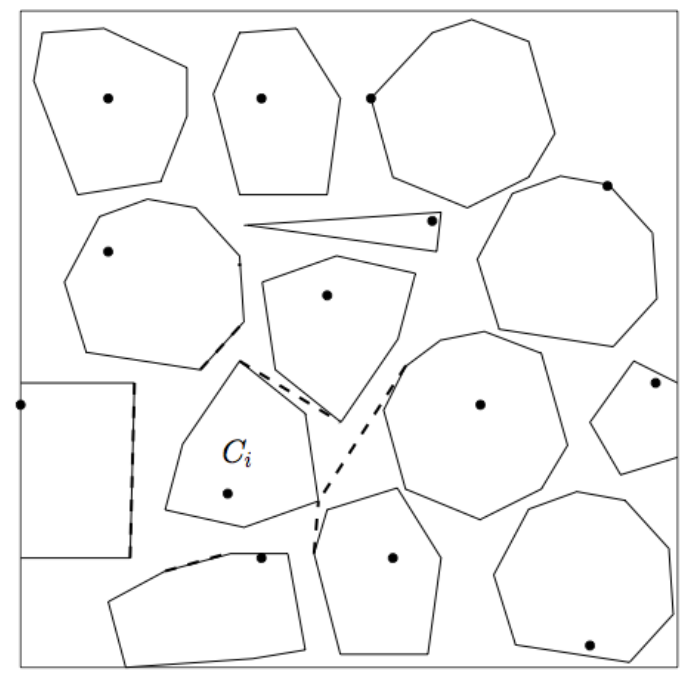

Figure 1. Non-overlapping minimum clique partitioning [12]

Definition 2.10: A PTAS(Polynomial Time Approximation Algorithm) is an algorithm which takes an instance of an optimization problem and a parameter $\epsilon>0$ and, in polynomial time, produces a solution that is within a factor $\epsilon$ of being optimal. For example, for an NP-Complete problem, a PTAS would produce an answer with size at most $(1+\epsilon) O P T$, with $O P T$ being the size of the optimal solution.

Pirwani's algorithm runs in $n^{O\left(\frac{1}{\epsilon^{4}}\right)}$ time. Dumitrescu et al [12] introduced a new PTAS for MIN-CLIQUE-PARTITION in UDGs with $n^{O\left(\frac{1}{\epsilon^{2}}\right)}$ running time.

Theorem 2.11: [12] There exists a PTAS for MCP(MAXCLIQUE-PARTITION) in unit disk graphs, which computes an $(1+\epsilon)$-approximation in $n^{O\left(\frac{1}{\epsilon^{2}}\right)}$ time.

They also proposed a randomized quadratic-time algorithm with approximation ratio 2.16 for this problem.

Theorem 2.12: [12] For any $0<\epsilon, \delta<1$ there is an algorithm for MCP in unit disk graphs, which computes a solution at most $1+\frac{2}{\sqrt{3}}+\epsilon$ times the optimal with probability at least $1-\delta$ in $O\left(\frac{1}{\epsilon} \cdot \ln \frac{1}{\delta} \cdot n^{2}\right)$ time. Inparticular, a $\frac{181}{84}$ approximate solution can becomputed with probability at least $1-\delta$ in $O\left(\ln \frac{1}{\delta} \cdot n^{2}\right)$ time.

All algorithms above search in the domain of the answers that are congurous with the property stated in theorem [15].

\section{Problem Formulation}

The basic problem of spectrum sensing is the detection of a primary user in a noisy environment. It is a difficult task especially with low SNRs due to fading and shadowing. The sensing problem can be characterized as a hypothesis test problem [8]:

$$
y_{t}= \begin{cases}n(t) & H 0 \\ h(t) s(t)+n(t) & H 1\end{cases}
$$

Where $y(t)$ is the received signal, $n(t)$ is the noise instance in time $t$ with variance, $s(t)$ is the transmitted signal that is 


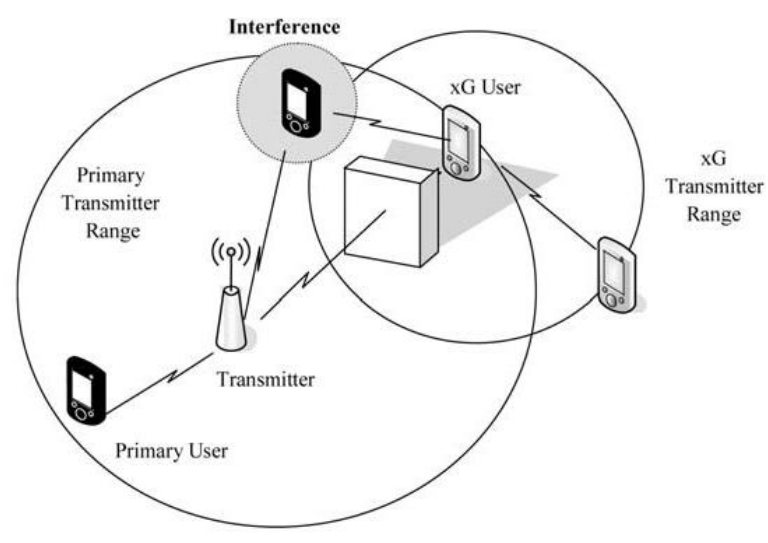

Figure 2. Hidden Terminal Problem [3], [7]

auto-correlated, $E\left\{|s(t)|^{2}\right\} \neq 0$, and $h(t)$ is the channel gain or attenuation. $\mathrm{HO}$ and $\mathrm{H} 1$ are the noise and signal presence hypotheses respectively. Classical techniques use the detected energy as an indicator for signal presence in the channel. The decision process is as follows [8]:

$$
\text { Decision }= \begin{cases}E\left\{|y(t)|^{2}\right\} \leq V_{T} & H 0 \\ E\left\{|y(t)|^{2}\right\}>V_{T} & H 1\end{cases}
$$

where $V_{T}$ is the variance of the noise. The energy often estimated by the sum below which is an imprecise estimator especially when a small number of samples are available [8]:

$$
E\left\{|y(t)|^{2}\right\} \approx \frac{1}{N} \sum_{k=1}^{N}|y(t)|^{2}
$$

Cooperative approaches for spectrum sensing use the information gathered by all receivers to determine the presence of a signal in a channel [4], [7]. This cooperation avoids the hidden terminal problem, in which the cognitive radio transmitter is unable to detect the primary transmitter due to shadowing or fading, but its transmission causes interference to the primary user transmission in the primary receiver. The scenario is depicted in figure III. Since the interference happens in receivers, it is possible to avid interference by primary receiver detection [4]. This method has shown practical only for TV receivers [9].

As it is obvious, cooperative spectrum sensing needs multiple sensors distributed over a large area. Its accuracy depends on the density of sensors over the area, since low density results in highly uncorrelated data received by sensors. Along with the density, information fusion technique used to combine the multi-source information and the decision making algorithm affects the performance of the scheme.

Consider a large area in which a primary user network is installed. For a cognitive radio network to take advantage of the white spaces throughout the primary user communication and avoid any interference to primary users, it is vital to draw a precise spectrum map.

The scenario depicted in III is as follows. Consider a primary network with fixed base stations that communicate

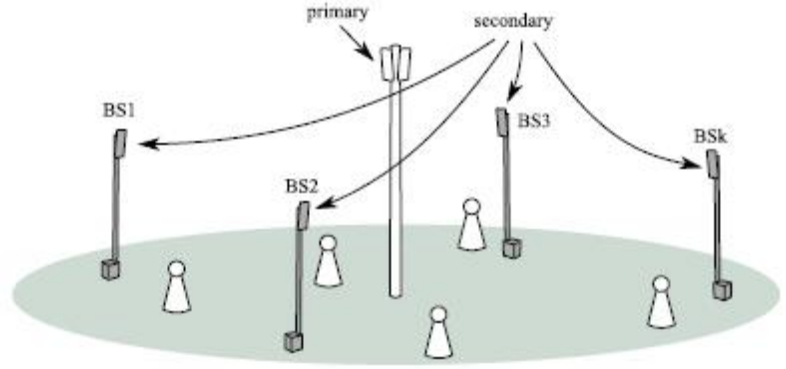

Figure 3. Sensing Scenario [8]

occasionally in a licensed band along with spatially random distributed spectrum sensing base stations of the cognitive radio networks distributed in a large region. The goal is to find the largest area in which no primary user transmission is detected. We call this are the white space region or WSR for short from now on. Then the cognitive radio users residing in the WSR exploit the opportunity to transmit to their neighboring cognitive radio users.

Assume that [8]:

- We have $K$ cognitive radio sensing base stations spread over the area randomly, but their precise spatial coordinates are known. Suppose that these base stations are able to communicate over a wired network and maintaining a control network is not of

- They all are able to sense the same portion of the spectrum.

We define $S$, the $K \times N$ sensing matrix to be defined by $N$ recently sensed samples by $K$ sensing base stations, where $y_{i}(k)$ is the $k^{t h}$ sensed sample by the $i^{t h}$ antenna:

$$
\mathbf{S}=\left(\begin{array}{ccc}
y_{1}(1) & y_{1}(2) & \ldots \\
y_{2}(1) & y_{2}(2) & \ldots \\
\vdots & \vdots & \ddots
\end{array}\right)
$$

In the case of $\mathrm{H} 0$, only noise present, the inner product of all rows will be an estimate of the noise autocorrelation function. Since it is assumed that noise samples are mutually uncorrelated, this value will be close to zero. We denote the $m^{t h}$ row of the matrix $S$ by $S_{m}$ we have:

$S_{m} \odot S_{n}=S_{m} S_{n}^{T}=\sum_{i=1}^{N} y_{m}(i) \times y_{n}(i) \approx N \sigma_{\text {noise }}^{2} \delta(m-n)$

where $\sigma_{\text {noise }}^{2}$ is the noise variance.

In case of $\mathrm{H} 1$, primary transmission available, the inner product of the rows will be proportional to the transmitted signals autocorrelation. We assume constant channel gain for the sensing period. As the noise is uncorrelated to the transmitted signal we will have: 


$$
\begin{gathered}
S_{m} \odot S_{n}=\sum_{i=1}^{N} y_{m}(i) \times y_{n}(i) \\
=\sum_{i=1}^{N}\left(\left(h_{n} . s(i)+N_{n}(i)\right) \times\left(h_{m} . s(i)+N_{m}(i)\right)\right) \\
\quad \approx h_{m} h_{n} \times N \times E\left\{|s(t)|^{2}\right\}(m \neq n)
\end{gathered}
$$

The goal here is to find some of submatrixes of S, called the Xsubmatrix, with all mutually uncorrelated rows and successive columns:

DEFINITION 3.1: Suppose that we have a $n \times m$ matrix with row set $A=\left\{r_{1}, r_{2}, \ldots, r_{n}\right\}$ and column set $B=$ $\left\{c_{1}, c_{2}, \ldots, c_{m}\right\}$. Xsubmatrix is the triple $(\Phi, p, q)$ where $\Phi \subseteq$ $A$ and $p \leq q$ is a submatrix formed by rows of the set $\Phi$ and columns of the set $\left\{c_{p}, \ldots, c_{q}\right\}$.

The Xsubmatrix identifies the adjacent antennas that receive no primary transmission. To form a WSR, we should take spatial information into account.

This paper has two parts. In the first part we consider the problem of finding the maximum XSubmatrix of matrix $S$. We will show that this problem is NP-Hard. Moreover We prove that this problem is also Hard to approximate. In the next part we take spatial information of antennas into account and propose a method for finding good WSRs.

\section{Finding the Maximum XSUbmatriX}

Now, we have a model of the problem suitable for using algorithmic techniques. A matrix with real entries, in which we want to find a XSubmatrix with maximum number of entries.

In this section we will show that the general setting of the problem is NP-Complete, means that there is no polynomial algorithm for it unless $P=N P$. From now we call this problem MXS(Maximum XSubmatrix). In the following theorem we will prove this fact. In order to prove this theorem we use the default approach. We must show that MXS is harder than an NP-Complete problem. To achieve this we should find an NP-Complete problem, $A$, reducible to MXS, means that we can find a polynomial algorithm for $A$, if there is a polynomial algorithm for MXS.

Theorem 4.1: The problem of finding the XSubmatrix wit maximum number of entries is NP-Complete(MXS is NPComplete).

Proof: We select the MAX-CLIQUE(definition 2.2) problem for our reduction process. Suppose that we have a graph $G$ and we want to find the maximum clique of it. Suppose $G$ has $n$ vertices $v_{1}, v_{2}, \ldots, v_{n}$ and $m$ edges $e_{1}, e_{2}, \ldots, e_{m}$. We construct a $n \times m$ matrix $M$ from $G$. The entries of $M$ will be defined in the following way:

$$
M_{i, j}= \begin{cases}1 & \text { if } v_{i} \text { is adjacent to } e_{j} \\ 0 & \text { otherwise }\end{cases}
$$

It is obvious that there are exactly two 1's in each column of $M$. Now consider the correlation of two rows of $M$. If the vertices $v_{i}$ and $v_{j}$ are connected in $G$ via an edge the dot product of row $i$ and row $j$ of $M$ will be 1 means that those rows are uncorrelated. In the other direction if there is no edge between $v_{i}$ and $v_{j}$ the dot product of those rows will be 0 and means they are correlated.

With the discussion above every clique of $G$ of size $k$ is related to $k$ uncorrelated rows of $M$. So if we had a polynomial algorithm for solving MXS, we could run it on $M$ constructed from $G$ and return its output as the indices of the vertices included in the maximum clique of $G$. This leads to contradiction because there is no polynomial time algorithm for MAX-CLIQUE problem unless $P=N P$.

It is trivial that we can reduce MXS problem to MAXCLIQUE problem and we can use the approximation algorithm proposed for MAX-CLIQUE for our problem without violating their factor. We call this type of reduction, L-Reduction. In the next two theorems we will prove that this problem is more harder than default NP-Complete problems.

So we can use the same proof we have used for theorem 4.1 to prove the following theorem:

Theorem 4.2: For each $\epsilon<1$ there is no approximation algorithm for MXS problem with factor $n^{1-\epsilon}$ if $P \neq N P$.

Proof: Since there is no approximation algorithm for MAX-CLIQUE problem with factor better than $n^{1-\epsilon}$ (from theorem 2.4) by using the same proof in theorem 4.1 this theorem can be obtained.

In the next section we will change the model according to cartesian coordinates of the antennas. We will see that finding spatial constrained cliques is not as hard as the general setting of the problem.

\section{USING SPATIAL INFORMATION}

In this section instead of looking to the whole matrix we consider Xsubmatrix that corresponds to a small connected convex region in which no primary transmission is detected. We do so by adding spatial information to the edge assignment policy. At first we will model the sensing matrix as a special graph that includes spatial information and then we will show the problem to be easier to solve in this new setting.

\section{A. Graph Modeling}

In this section we propose a method to find a WSR from the matrix $S$ modeled as a Unit Disk Graph (UDG). We model the matrix with a graph $G=(V, E)$ where each vertex in $V$ corresponds to a sensing base station. An edge is assigned to a vertex pair if their sensed signals are uncorrelated (the inner product of their corresponding rows yield a value near zero). We define the threshold for their correlation value to be the noise covariance. To take the spatial information into account, we also define a distance threshold for vertex pairs to receive an edge. By this means we form a partial UDG graph in which not only the distance of two ends of an edge is below a threshold, but also their correlation value is below the noise variance.

We use this graph to find a WSR. A WSR is formed of some locally distributed antennas that receive no primary transmission. Transforming the problem to the graph world, we should find the sufficiently large complete subgraphs. This problem tends to be the MAX-CLIQUE(definition 2.2) 
and MIN-CLIQUE-PARTITION(definition 2.3). This complete subgraph represents a set of mutually uncorrelated antennas that are locally distributed over a small subregion. Once the clique is formed, we find the convex hull of the vertices. We then inform the cognitive radio users residing inside the convex hull to use the white space found, but limiting their transmission to remain inside the borders of the convex hull. For simplicity we assume that due to simple channel propagation model [14] the probability of a base station receiving primary transmission inside the convex hull is low (because of small surface area of the convex hull). However any clique containing a receiving base station could be detected by a simple algorithm and removed.

\section{B. Finding the Regions}

In this new model we deal with a UDG in which we have removed some of the edges. In this section we call this UDG, $G$. In the new problem setting we still deal with finding cliques. As we stated in the previous subsection each clique in $G$ has a good chance to be a clique in $H$ too.

In the first approach we exploit the method proposed by Clark et al [13] stated in theorem 2.7 to find the largest subregion that receives no primary transmission that is a maximum clique of $G$. There should not be any base station receiving transmission inside the output clique, Therefore we check all sensing base stations inside the convex hull formed of clique vertices each time to see if they receive any primary transmission. In this algorithm we increase the UDG threshold at each step to find a clique with the maximum area. The details of the algorithm are expressed in algorithm 1 .

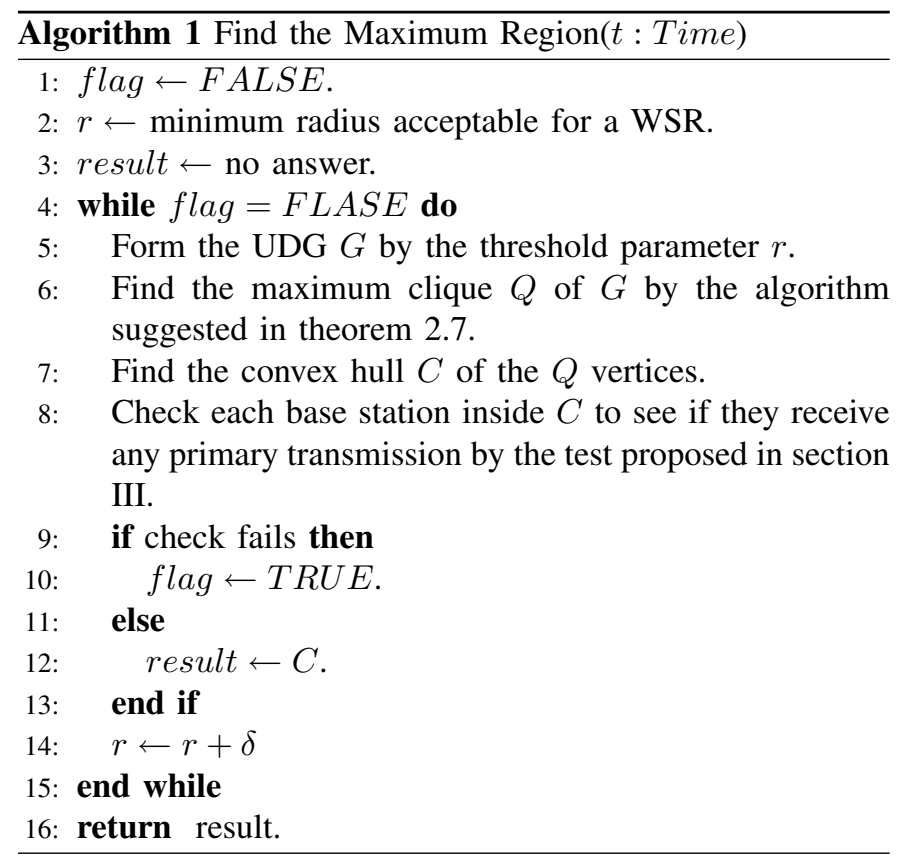

In figure V-B you can see the mechanism of algorithm 1. In this figure correlated and uncorrelated antennas are shown by black and white circles respectively. In the first stage algorithm starts by setting $r=r_{\min }$ and finds the maximum

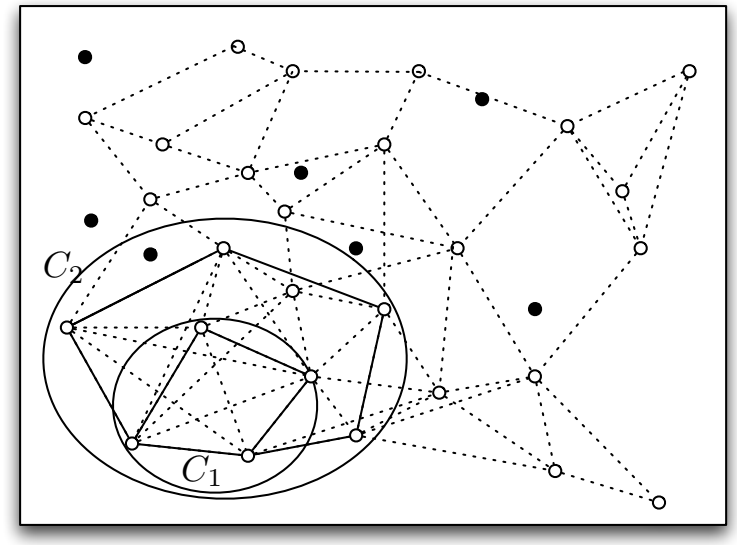

Figure 4. $C_{1}$ is the maximum region formed by $r=r_{\min }$ in algorithm 1. $C_{2}$ is the maximum region in the second stage of algorithm 1 where $r=r_{\min }+\delta$

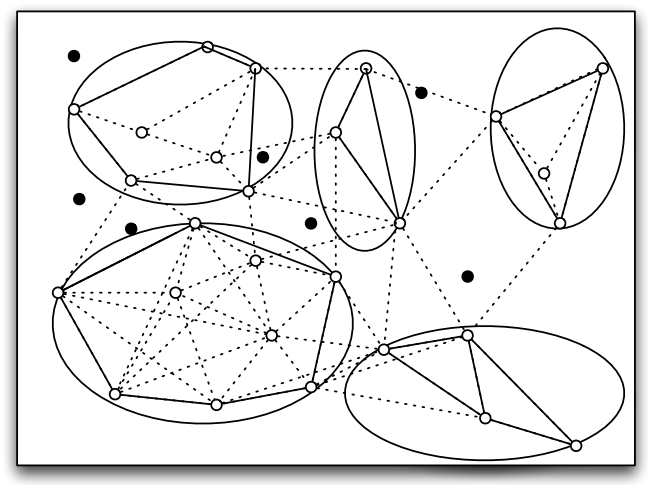

Figure 5. Output of algorithm 2

clique convex hull in the UDG formed by this threshold. The algorithm finishes in the second round where $r$ has been set to $r_{\text {min }}+\delta$.

In the second approach we use the algorithms proposed for MIN-CLIQUE-PARTITION problem for UDGs. In this approach at first we find the partition of graph $G$ into minimum number of cliques. Find the convex hull of them, and check their validity during the time as we do in the first approach. We must emphasize that the output of algorithms in [12] follow the conditions of the following theorem through which they tend to have non-overlapping convex hulls. To have regions with more area, after the regions have found, we increase the UDG threshold for each of them independently while they remain valid and then we extend the area in which they reside. You can see the details of this approach in algorithm 2

In figure V-B we show the style of algorithm 2's output.

\section{CONCLUSION}

In this paper we have proposed a new scheme for cooperative spectrum sensing. We have exploited the spatial 


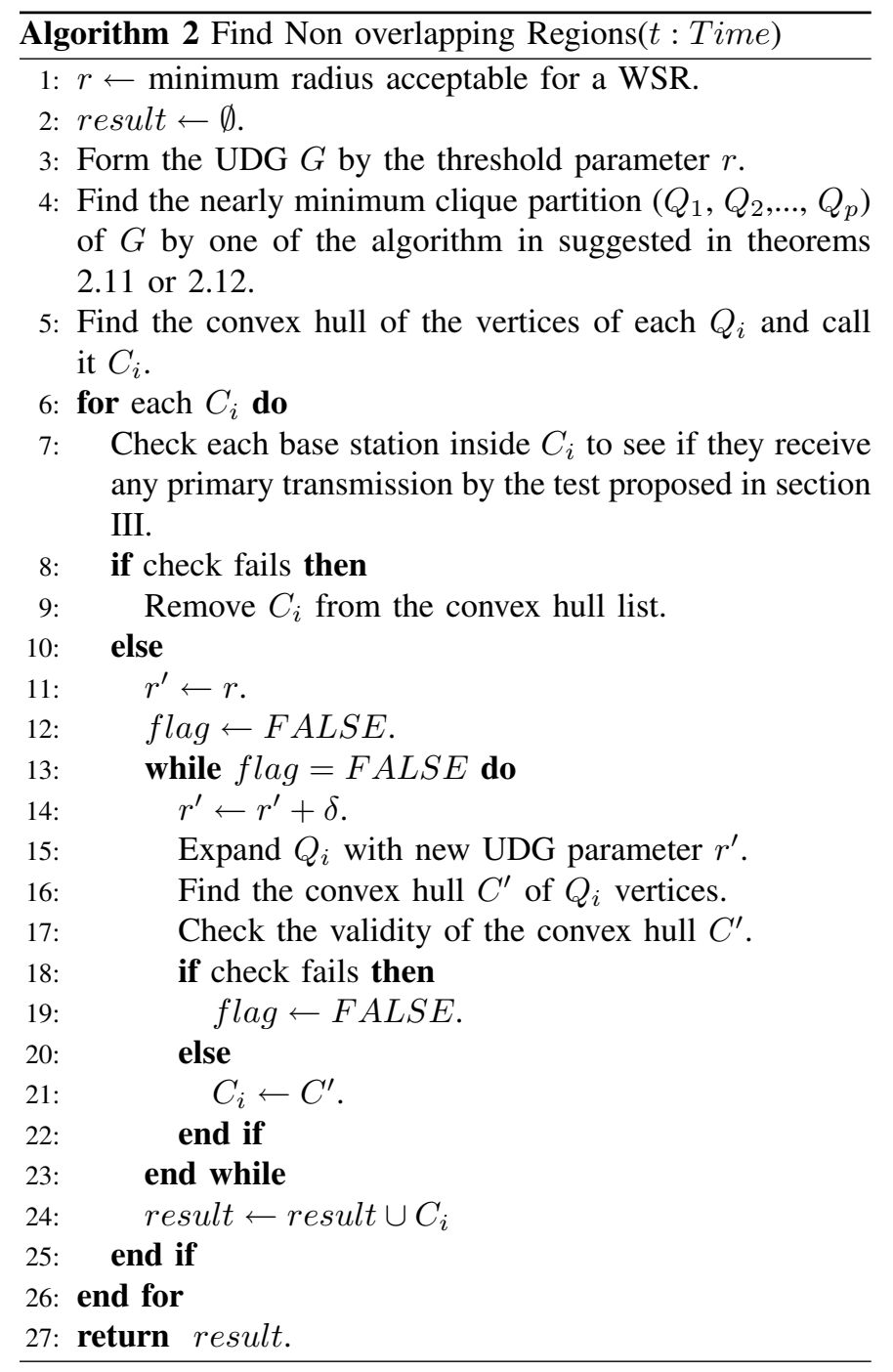

information of the antennas to find spectrum holes inside local regions. We have modeled our problem setting as a special kind of graphs called UDG. In the new setting our problem tends to be much like MAX-CLIQUE and MIN-CLIQUEPARTITION problem that are NP-Hard in general, but are solvable in polynomial time in our setting. Our method can be enhanced by taking direction of the edges into account to find the largest subregions possible.

\section{REFERENCES}

[1] Federal Communications Commission, Notice of proposed rule making: Unlicensed operation in the TV broadcast bands, ET Docket No. 04-186 (FCC 04-113), May 2004.

[2] I. Mitola, J. and J. Maguire, G. Q., Cognitive radio: making software radios more personal, IEEE Personal Commun. Mag., vol. 6, no. 4 , pp. 1318, Aug. 1999.

[3] I. F. Akyildiz et al., NeXt Generation/Dynamic Spectrum Access/Cognitive Radio Wireless Networks: A Survey, Comp. Networks J., vol. 50, Sept. 2006, pp. 212759.

[4] I. F. Akyildiz, W.Y. Lee, M. C. Vuran, and S. Mohanty, A survey on spectrum management in cognitive radio networks, IEEE Commun. Mag., vol. 46, pp. 4048, Apr. 2008

[5] S. Haykin, Cognitive Radio: Brain-Empowered Wireless Communications, IEEE JSAC, vol. 23, no. 2, Feb. 2005, pp. 20120.
[6] D. Cabric, S. M. Mishra, and R. W. Brodersen, Implementation Issues in Spectrum Sensing for Cognitive Radios, Proc. 38th Asilomar Conf Sig., Sys. and Comp. 2004, Nov. 2004, pp. 77276.

[7] T. Yucek and H. Arslan, A Survey of Spectrum Sensing Algorithms for Cognitive Radio Applications, IEEE Communications Surveys \& Tutorials, 2008, in press.

[8] L. S. Cardoso, M. Debbah, P. Bianchi, J. Najim, Cooperative spectrum sensing using random matrix theory, IEEE ISWPC, pp. 334338, May 2008.

[9] B. Wild and K. Ramchandran, Detecting Primary Receivers for Cognitive Radio Applications, Proc. IEEE DySPAN 2005, Nov. 2005, pp. 12430 .

[10] J.Håstad, Clique is hard to approximate withinn 1- $\varepsilon$, In Proc 37th IEEE Symp on Foundation of Computer Science, 627-636, 1996.

[11] I. Pirwani and M. Salavatipour, A PTAS for minimum clique partition in unit disk graphs, preprint, arXiv: 0904.2203, 2009.

[12] A. Dumitrescu, J. Pach, Minimum clique partition in unit disk graphs, CoRR abs/0909.1552, 2009

[13] B.N. Clark, C.J. Colbourn, and D.S. Johnson, Unit disk graphs, Discrete Mathematics, 1990.

[14] A.J.Goldsmith, Wireless Communications, Cambridge University Press, 2005.

[15] V. Capolyleas, G. Rote, and G. Woeginger: Geometric clusterings, Journal of Algorithms, 12 (1991), 341356.

[16] D. Zuckerman: Linear degree extractors and the inapproximability of max clique and chromatic number, Theory of Computing, 3(1) (2007), 103128.

[17] K. J. Supowit: Topics in Computational Geometry, PhD Thesis, University of Illinois at Urbana-Champaign, Report UIUCDCS-R-811062, 1981.

[18] TH Cormen, CE Leiserson, RL Rivest, Introduction to Algorithm, 2nd edition, The MIT press, 2001. 